of which was covered with a dirty grey-coloured eschar. Serum from vesicles contained a few bacilli; half a drop, given to a rabbit, had no perceptible effect. 9 P.M. He had no pain or distress. The tongue was moist. He had taken food moderately. The swelling harl extended over the scapula; as far as the middle, it was hard, terminating by a distinct ridge; beyond, it was softer. In front, the swelling had extended over the chest, and was not so hard as behind. Pulse II6, soft. After he had been helped up in bed to the sitting position, and lay down again, the pulse was 130 , and irregular. Temperature in the left axilla $99.8^{\circ}$, in the rectum $104.1^{\circ}$.; respiration 24 .

May 24th, I0.30 A.M. He passed a fairly good night; was not restless. He had no pain, but some uneasiness over the abdomen, which was relieved by vomiting. $\mathrm{He}$ appeared calm and composed. Pulse 130, very small, and difficult to count. Temperature in the left axilla $99.1^{\circ}$, in the right axilla $99.8^{\circ}$, in the rectum $102.3^{\circ}$; respiration 22. Serum from vesicles contained ten to twenty bacilli in each field. The contents of two tubes, given to a mouse, were fatal in seventeen and a half hours. 9.30 P.M. He had a restless day; passed no urine; his appetite was gone; his face shrunken. The swelling was extending; the extremities were cold; pulse imperceptible; temperature under the tongue $95^{\circ}$. He died ten hours afterwards. Serum from the arm contained bacilli ; two tubes, given to a mouse forty-five hours afterwards, were fatal in sixty-five hours.

Post mortem examination, made by Mr. Kenyon, nine hours after death. The ends of the fingers were of a dark colour. The upper surface of the scrotum had a dark-red colour. The swelling of the arm was much more yielding than during life. The injured part of the forearm was removed; the cut surfaces were crowded with small interstitial hæmorrhages. There were. no pleural effusions, nor enlargement of bronchial glands. The blood was fluid. There was no staining of the endocardium; no subserous petechial spots in the heart. All the viscera were apparently natural. Portions of the internal organs were sent to Professor Greenfield for examination. Serum from the arm contained numerous long motionless bacilli ; after keeping two days in a capillary tube, the bacilli were very long and convoluted.

\section{WOOLSORTERS' DISEASE.}

\section{By S. LODGE, M.R.C.S., Bowling, Bradford.}

JOSEPH BOOTH, aged 62, had suffered from bronchitis several times. He was inconvenienced by hydrocele, and this I had tapped twice or thrice, at distant intervals. He was a somewhat weak, but temperate and regular workman, and found constant employment as a comber, washer, or carder of wool. He was engaged in the latter occupation up to May 4th, and he died early on the 6th.

On the 5 th, a message was received from him of the usual character-not a particularly pressing one-and Mr. McArthur visited him. He found him sitting upright in bed, quite sensible, breathing rather quickly but easily; he complained of pain in the head and in the region of the liver; he had vomited some dark fluid. The tongue was furred, and red at the margins; the temperature low; pulse small and quick; the skin moist; and he was blue all over, but especially upon the extremities, both upper and lower. There was great congestion of the lungs, but no rales could be detected. The unfortunate man died after thirty hours' illness.

With respect to the certificate, we agreed that it would be safest not to assign a first cause of death; but, as the second, in Mr. McArthur's absence, I wrote " venous congestion".

For important sanitary reasons, it was necessary to have the body removed as soon as possible; but, before the interment, I determined, as far as circumstances permitted, to ascertain the post mortem condition: and, therefore, forty-eight hours after death, made a second external inspection of the body, and secured a few tubes of blood from the jugular vein. I had previously mentioned the case to Dr. Bell, and I believe he did the same.

Immediately after death, there was discoloration, almost amounting to blackness, over the face, neck, and chest ; but, on my second inspection, when the blood was taken, this appearance had considerably changed; still there was much lividity of these parts, and of the extremities also.

The blood was immediately handed to Mr. Tacey for examination, and he reports having found a few rods, i.e., bacilli, and, when the fluid was stained (according to Kock's method) with aniline violet, some spores. His observations were made with a Zeiss, $16 \times 1500$ diameters.
The remarkable point in connection with the foregoing case is, that the man was taken ill when employed at a carding machine. The material he was watching, as it passed through the process, had been previously twice washed at a temperature of $130^{\circ}$, and afterwards dried in a swift current of hot air. Of course, it had been carried through the preparing machines (five in number), and twice combed. In fact when carded, it only required to be rolled into balls to finish it.

\section{SIX CARDIAC AND VASCULAR CASES: WITH REMARKS AND ENGRAVINGS.}

By E D W I N R I C K A R DS, M.A., M.D., Physician to the General Hospital, Birmingham.

CASE 1. Stenosis of the Pulmonary Valve: Imperfect Ventricular Septum: Malposition and Narrowing of the Pulmonary Artery: Narrowing of the Descending Part of the Arch of the Aorta.-John L., aged 2r, was an out-patient at the General Hospital, January 1876. He was cyanotic; his lips, ears, fingers, and toes were of a purple hue; his skin was thin; his fingers bulbous; his nails incurved. From his account, there did not appear to be any family tendency to disease or malformation of the heart, rheumatism, syphilis, or disease of the lungs. He earned his livelihood by playing a whistle in the streets. All his life he had been blue-skinned, and subject to attacks of palpitation of the heart, accompanied with cough, shortness of breath, and pain in the chest, the pain extending from nipple to nipple. He was especially liable to these attacks at the end of a long tune and in cold weather. When he was so affected, he said, he became "as black as a coal, and his veins stood out". His hands and feet were always cold. He was fully developed, though somewhat undersized for his age. He was very intelligent. His chest was carefully examined. There were no signs of disease of the lungs; no marked increase of cardiac dulness; no thrill. The apex-beat and impulse were normal. A coarse superficial systolic murmur was heard at the base of the heart, having its maximum intensity at the second left costal cartilage. Between the base and apex of the heart was heard another murmur, soft and systolic, and having its maximum intensity over the sternum on a line with the nipples. At the apex of the heart, the two sounds were distinct, though the first was accompanied by a soft systolic murmur. The pulse, 80 per minute, presented no peculiarity; the temperature in the mouth was $97.4^{\circ}$; the urine was not albuminous.

In consequence of notes being taken of his case by Mr. Spofforth (the physicians' assistant), and of some remarks I made to the students, he was lost sight of until the following December, when he was admitted into the hospital with acute phthisis, of which he shortly died. Between his being an in- and an out-patient, he had three attacks of profuse hæmoptysis. At no period was there any dropsy.

A post mortem examination was obtained with difficulty, and was. made under rigid conditions. The lungs were studded throughout with small cheesy masses of catarrhal pneumonia, some of which had softened. There were a few small cavities. The heart was slightly enlarged, and weighed ten ounces and a half; its muscle was healthy. The aorta was much narrowed beyond the origin of the left subclavian artery. The pulmonary artery was very narrow, and was situated on the right side of the aorta. The right chambers of the heart were dilated, and their walls somewhat thickened; the left chambers appeared of normal size. The septum ventriculorum was deficient at its base, so that the finger could be passed from the right to the left. ventricle, and from the right ventricle into the aorta. The pulmonary valve was represented by a conical diaphragm, with a central aperture scarcely large enough :o admit of the passage of a crow-quill; in this. diaphragm were three deep indentations, showing its origin from the three segments of the pulmonary valve. The auriculo-ventricular and aortic valves were perfect; on all of them were small vegetations, evidently of long standing. The aorta appeared to come off from both ventricles equally. The foramen ovale and ductus arteriosus were both closed. The engraving shows the right ventricle opened along its right border; its anterior part removed; the pulmonary artery turned back and drawn over to the left side, so that the posterior part of that vessel is seen anteriorly in the engraving. A slit has been made in the posterior part of that vessel to expose the conical diaphragm, through which a fine curved probe has been passed. The left ventricle has been incised, and a thick probe passed from it through the abnormal aperture in the septum into the right ventricle. A slit has been made in the anterior part of the aorta. The small vegetation are only apparent on the tricuspid valve, and there indistinctly. I am 
indebted to Dr. Philip Bindley for the drawing of the specimen from which the engraving was made.

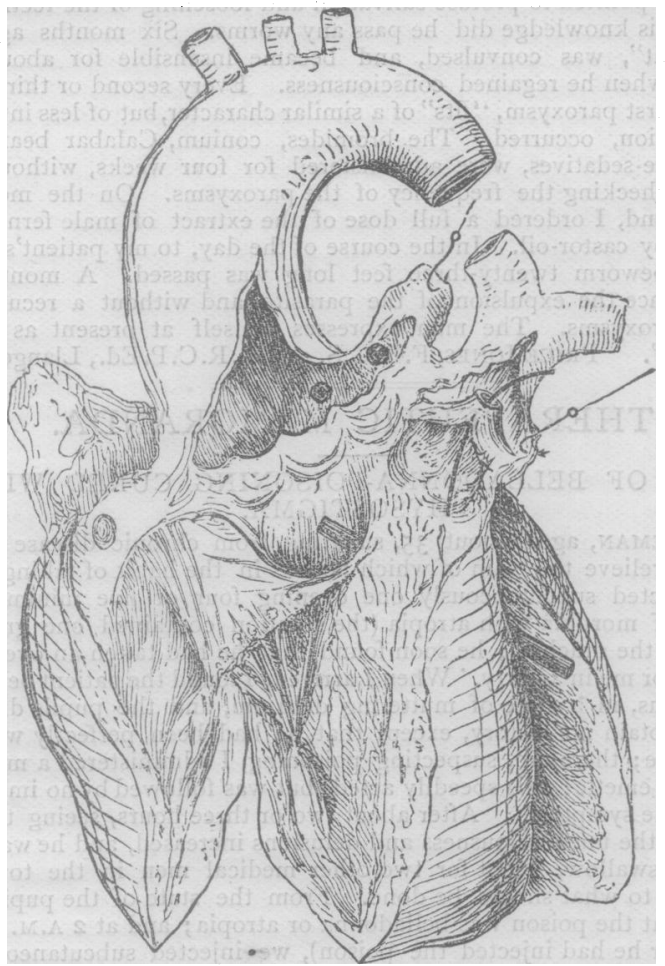

REMARKS. - The record of this case may be useful for the purpose of statistics, and so help to throw light on the origin of perhaps the most common form of malformations of the heart-that of imperfect ventricular septum, associated with stenosis of the pulmonary valve. It also exemplifies other abnormalities of less frequent occurrence.

I am an advocate of the view that, in a heart where the ventricular septum is found imperfect and the pulmonary valve narrowed, the former condition is the result of the latter; that the pulmonary stenosis exists before the septum, under ordinary circumstances, would be complete; and that, this being the case, the blood in the right ventricle, not finding a free exit by the pulmonary artery, escapes into the left ventricle, and, by continuing to do so, prevents the closure of the septum. As to the origin of the stenosis of the pulmonary valve in such cases, I agree with those who think that it is generally, if not always, due to a fault in development rather than to disease; for, assuming the imperfect septum to be due to the pulmonary stenosis, from the frequent association of the two conditions, intra-uterine endocarditis would appear to be far more common before the end of the third month of fotal life than afterwards, since it is at that time (say the embryologists) that the septum is perfected. In the case recorded, the segments of the pulmonary valve, had they become adherent from inflammation, would probably, by the fluxion of time, have been so altered and fused together that the origin of the conical diaphragm from three segments would not have been discernible. The presence of small vegetations on the otherwise healthy valves of the heart might be urged in favour of the amalgamation of the segments of the pulmonary artery being due to an intra-uterine endocarditis; but these warty growths may have been produced after birth, and in consequence of abnormal tension of the endocardium, and from obstructed circulation through the heart. The abnormality in the circuit of blood through the heart, occurring at such an early period of embryonic life, may, by pressure, have caused a disturbance of the normal relations of the great vessels, and thus the malposition of the pulmonary artery may be accounted for. The balancing power in the animal economy, by which physiologically the demand of blood to a part regulates the supply, may fairly be supposed to be in force during development, and to determine the size of a vessel by the amount of blood it will have to carry. By the acceptance of such a hypothesis, an explanation is afforded of the narrowing of the pulmonary artery and aorta. The stenosis of the pulmonary valve would limit the supply of blood to the pulmonary artery, and so to the ductus arteriosus; which in foetal life supplies with blood that part of the aorta beyond the origin of the left subclavian artery, or, in other words, that part of the aorta which in this case is narrowed. Dr. Wickham Legg, in the record of a case of complete obliteration of the aorta at the point of insertion of the remains of the ductus arteriosus (Trans. Path. Society of London, vol. xxix), expresses the opinion that narrowing of the aorta at that point is due to changes which take place after birth in the ductus arteriosus. Dr. Peacock (British and Foreign Med.-Chir. Review, vol. xxv) urges that the defect is often associated with other congenital malformations, and argues that it is the result of faulty development of the branchial arches which form the continuation of the aorta from the origin of the left subclavian to beyond the insertion of the ductus arteriosus. The dilatation of the right side of the heart, and the great distension of the superficial veins of the body when the patient whistled, seem to support the view that, in the causation of the cyanosis, the imperfect oxidation of blood and the mixture of venous with arterial blood were minor factors, compared with systemic venous congestion. The age attained by John L. was in excess of that usually reached by the subjects of such malformations; and it is noteworthy that death was caused by disease of the lungs with hæmoptysis in one in whom there appeared to be no hereditary tendency to pulmonary disease. The lowered vitality of those organs, in consequence of defective circulation through them, doubtless predisposed them to degenerative disease.

\section{SURGICAL MEMORANDA.}

\section{A NEW METHOD OF TREATING RECTAL FISTUL}

ABOUT three months ago I tried a plan of operating in a case of fistula in ano, which I believe to : novel-if there be anything new-and as the result was very satisfactory and rapid, I venture to draw attention to it, although I have only one case, so far, to record. The fistula was about two and a half inches long, and was divided in the usual way, then all pseudo-membranous tissue and granulations were scraped away with a sharp scoop, and the ordinary narrow bluntpointed bistoury, buried for half its depth at the fibre of the fistula. Both surfaces of the wound being vivified and hæmorrhage having ceased, three deep silver s.tures were passed, entering the skin about a quarter of an inch from the edge of the wound, and, passing well beneath the floor of the fistula, were brought out at the same distance on the other side of the wound. The upper stitch ran through the coats of the rectum into the surrounding tissue. These stitches were removed a week after the operation (but might have been removed sooner) when the wound was soundly healed. The patient has been perfectly well since. I intend to give this plan an extensive trial, as it promises to considerably expedite the cure of suitable cases of rectal fistula, and to do away with the painful dressings which have to be resorted to in the common mode of treating such affections. The bowels should be well opened by a purgative and enema before operating, and must be kept confined for about four days after the operation. All loose folds of skin about the fistulous track must be removed. I shall be glad if other surgeons will try this plan.-I am, etc. H. A. REeves, F.R.C.S. Assistant Surgeon to the London Hospital, 6, Grosvenor street, W. Surgeon to the Hospital for Women, etc.

\section{OBSTETRIC MEMORANDA.}

\section{LACERATIONS OF THE CERVIX UTERI.}

THE modern gynæcological views of the frequency and importance of cervical laceration, during the process of parturition, require serious and impartial criticism. There are thousands of experienced and successful obstetricians in this country who have never recognised the condition even as a cause of serious hæmorrhage, and much less as necessitating an immediate complex operation, or being the occult complement of a chronic invalidism. In my experience of over two thousand labours, three-fourths of which were plebeian, no case of cervical laceration occurred which necessitated anything beyond a kite-tail temporary plug of dry cotton-wool, in addition to the usual every-day resources of cold, quiet, elevation of the pelvis, and bandage. Trickling hæmorrhage, with a firmly contracted uterus, may arise from anterior vulval, vaginal, or uterine tearing ; and, when the locality is sought with eye, hand, or speculum, or by their combination, heat, cold, elevation, or temporary pressure will usually suffice to arrest it. As, however, a primiparous case is known to me, in which post partum hæmorrhage from a retro- 